Supporting information for:

\title{
Ammonia Recovery from Domestic Wastewater Using a Proton-mediated Redox Couple
}

Weikun Chen ${ }^{1}$, Stefan Grimberg ${ }^{1,2}$, Shane Rogers ${ }^{1,2}$, and Taeyoung Kim ${ }^{1,3^{*}}$

${ }^{1}$ Institute for a Sustainable Environment, Clarkson University, Potsdam, NY 13699, United States

${ }^{2}$ Department of Civil and Environmental Engineering, Clarkson University, Potsdam, NY 13699, United States

${ }^{3}$ Department of Chemical and Biomolecular Engineering, Clarkson University, Potsdam, NY 13699, United States

*Corresponding Author: tkim@clarkson.edu

Number of pages: 14

Number of figures: 11

Number of tables: 1 


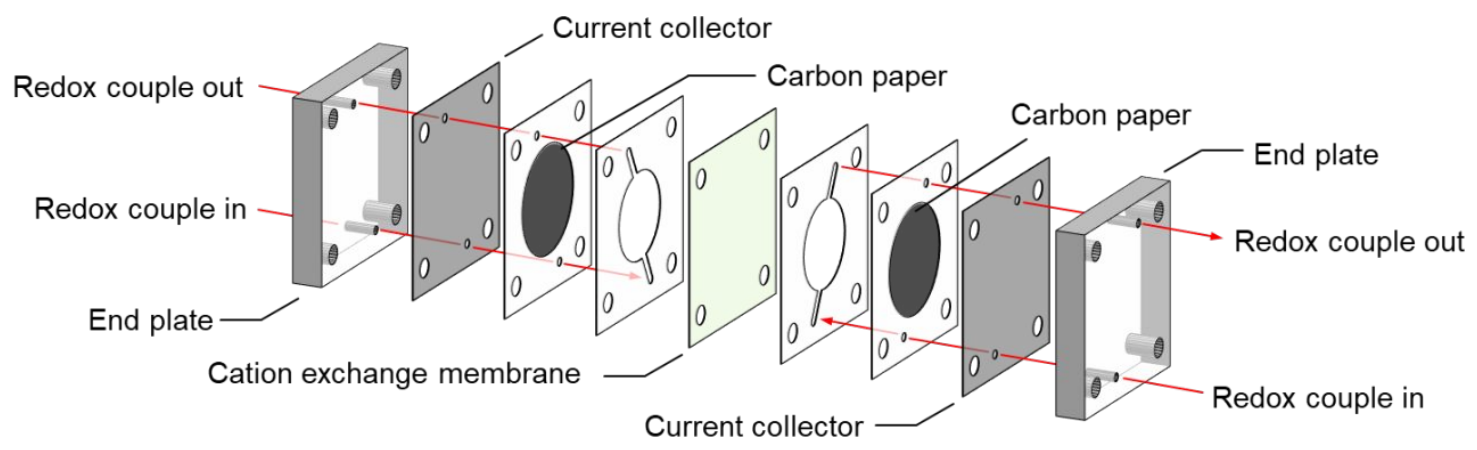

Figure S1. Construction of the custom-built 2-channel electrochemical flow cell.

(a)

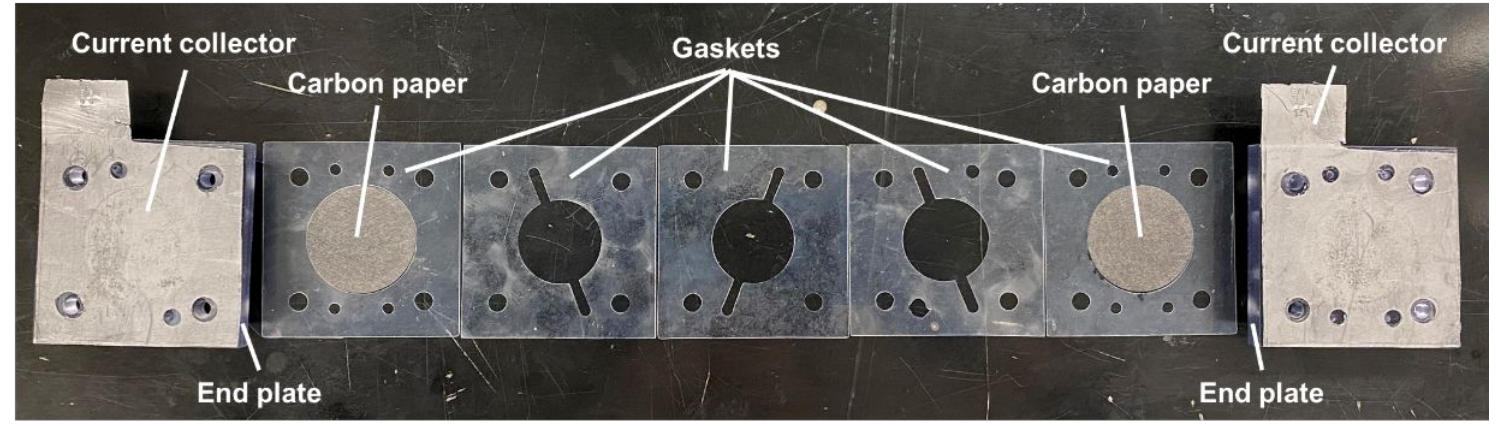

(b)

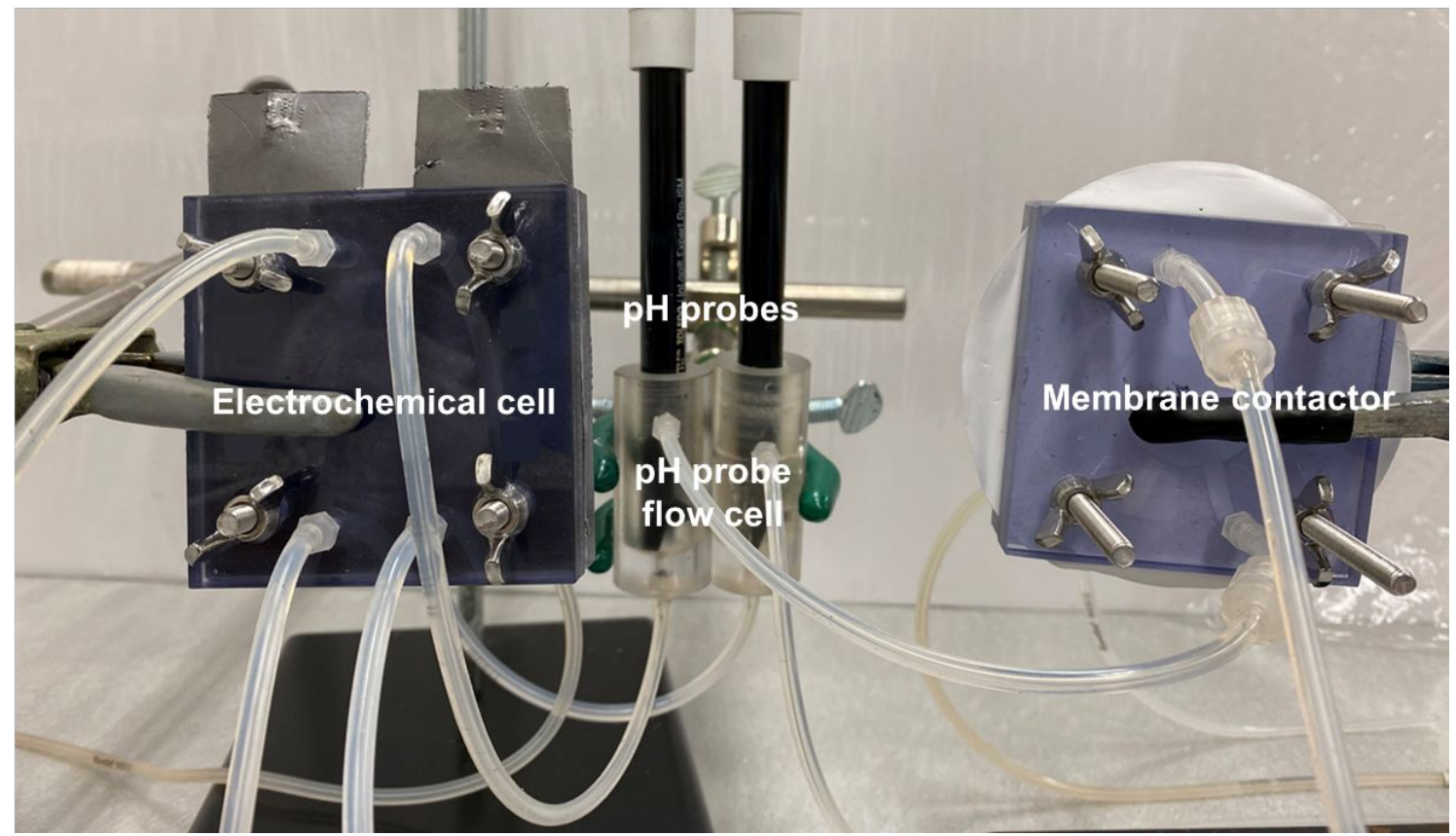

Figure S2. Photos of (a) custom-built 3-channel electrochemical flow cell compartments and (b) overall system. Spacers and cation exchange membranes are not shown here. 

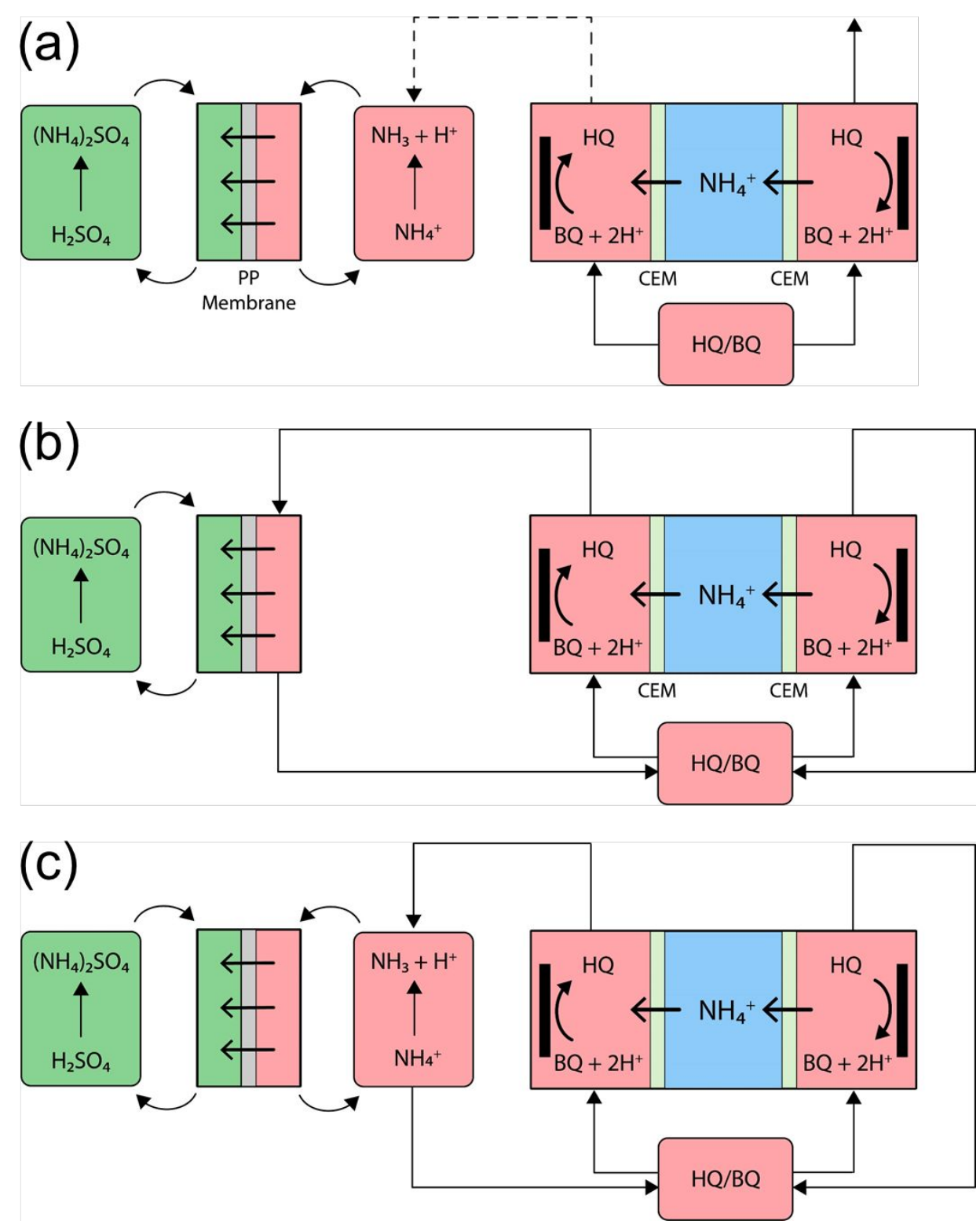

Figure S3. Schematic of the membrane contactor operated in (a) a batch mode using the cathode effluent collected in advance, (b) a single-pass mode using cathode effluent directly fed to the membrane contactor, and (c) a continuous mode using an equalization tank to hold a small volume of the cathode effluent, which was continuously fed to the membrane contactor. PP membrane: polypropylene membrane. 
(a)

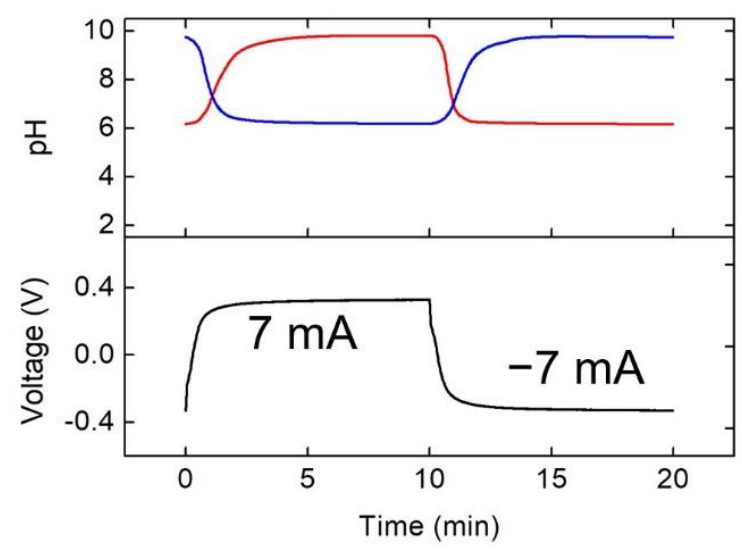

(b)

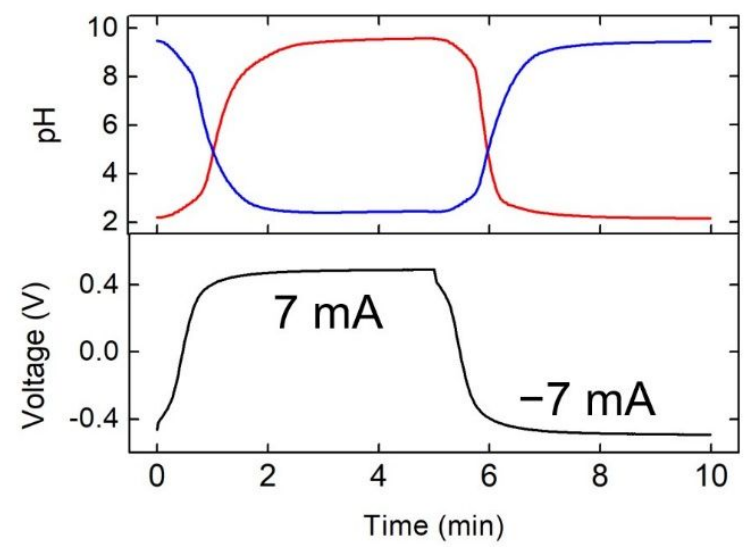

Figure S4. Effluent $\mathrm{pH}$ and voltage profiles recorded at $\pm 7 \mathrm{~mA}$ in the 2-channel flow cell at a flow rate of $0.5 \mathrm{~mL} \mathrm{~min}^{-1}$ by feeding each channel using (a) $15 \mathrm{mM} \mathrm{HQ} / \mathrm{BQ}+0.2 \mathrm{M} \mathrm{CaAc}$ and (b) $15 \mathrm{mM} \mathrm{HQ} / \mathrm{BQ}+0.2 \mathrm{M} \mathrm{CaCl}_{2}$.
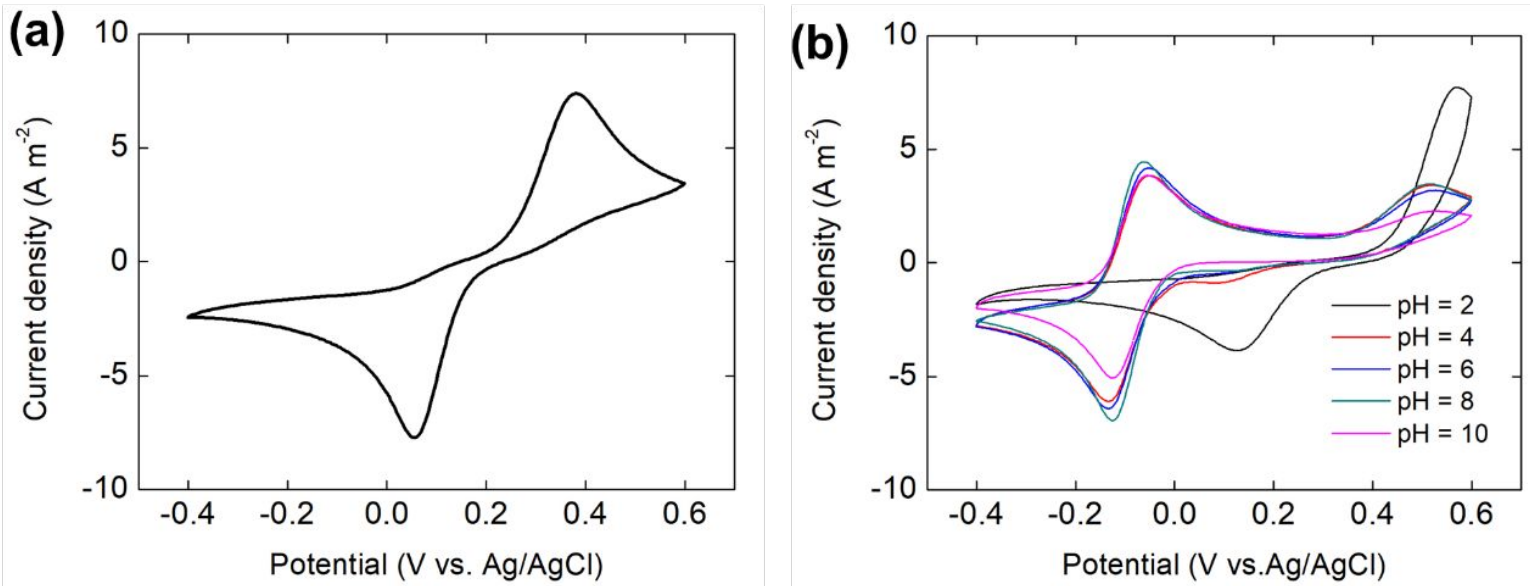

Figure S5. Cyclic voltammetry profiles of (a) $1 \mathrm{mM} \mathrm{HQ} / \mathrm{BQ}+0.2 \mathrm{M} \mathrm{CaAc}(\mathrm{pH}=4.7)$ and (b) 1 $\mathrm{mM} \mathrm{HQ} / \mathrm{BQ}+0.2 \mathrm{M} \mathrm{CaCl}_{2}(\mathrm{pH}=2$ to 10$)$ recorded in a 3-electrode cell using working (glassy carbon), counter (platinum wire), reference $(\mathrm{Ag} / \mathrm{AgCl}$ in $3 \mathrm{M} \mathrm{NaCl})$ electrodes at a scan rate of $100 \mathrm{mV} \mathrm{s}^{-1}$. The peak anodic current shifted to a more positive potential at a low $\mathrm{pH}$ created during the operation, supporting that a larger cell voltage in an unbuffered solution $\left(\mathrm{CaCl}_{2}\right)$ led to a relatively high cell voltage compared to a buffered solution ( $\mathrm{CaAc})$. 

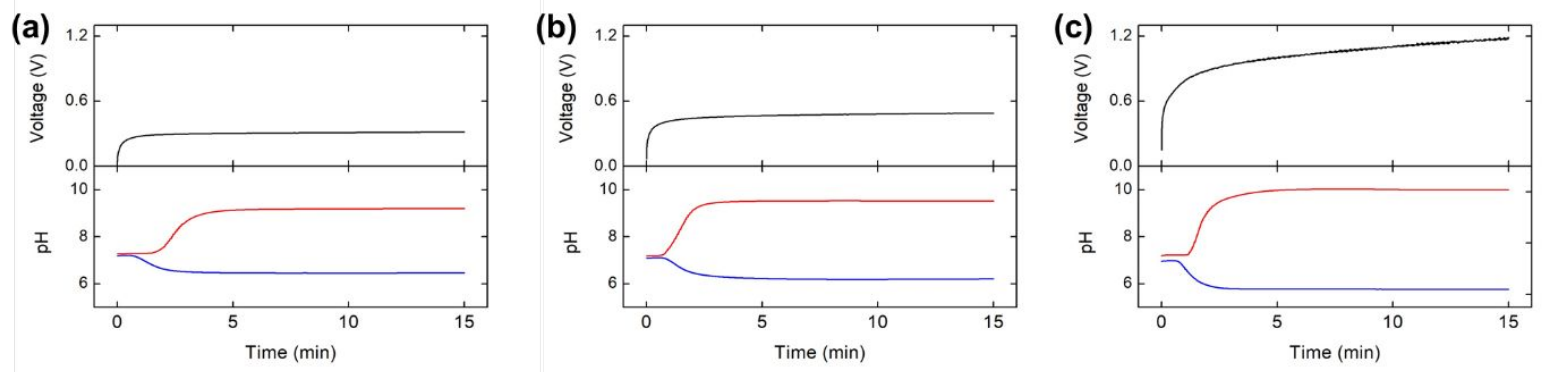

Figure S6. Representative voltage (top) and effluent $\mathrm{pH}$ (bottom) profiles recorded at (a) 4.8, (b) 9.6, and (c) $19.2 \mathrm{~A} \mathrm{~m}^{-2}$ using the 3-channel electrochemical flow cell. A redox couple (15 mM $\mathrm{HQ}, 15 \mathrm{mM} \mathrm{BQ}, 0.2 \mathrm{M} \mathrm{CaAc}$ ) was fed to anode and cathode channels and a synthetic wastewater $\left(20 \mathrm{mM} \mathrm{NaCl}, 5 \mathrm{mM} \mathrm{NH}_{4} \mathrm{Cl}, \mathrm{pH}=8.5\right)$ was fed to the central channel all at a flow rate of $0.5 \mathrm{~mL}$ $\min ^{-1}$. 


\section{Supporting Information Note 1. Evaluating diffusional exchange}

The 2-channel flow cell (Figure S1) was used to investigate diffusional exchange of salts between each channel. The two channels were divided by a regular CEM or modified CEM using a layerby-layer technique as described in the manuscript. The modified CEM was shown to provide a high monovalent ion selectivity in previous studies. ${ }^{1,2}$ Synthetic wastewater $(20 \mathrm{mM} \mathrm{NaCl}+5 \mathrm{mM}$ $\mathrm{NH}_{4} \mathrm{Cl}$ ) and $0.2 \mathrm{M} \mathrm{CaCl}_{2}$ (or $0.2 \mathrm{M} \mathrm{NaCl}$ ) solutions were circulated through each side of flow cell at a flow rate of $0.5 \mathrm{~mL} \mathrm{~min}{ }^{-1}$ without recycle. Ammonium transport through a CEM due to diffusional exchange between $\mathrm{Ca}^{2+}$ or $\mathrm{Na}^{+}$was quantified using a spectrophotometer (Hach DR 900, standard method 10031). The use of modified CEM reduced the diffusional exchange between $\mathrm{NH}_{4}{ }^{+}$and $\mathrm{Ca}^{2+}$ by $84 \%$ compared to regular CEM (Figure S7). An additional experiment confirmed that the reduced exchange was due to the presence of $\mathrm{Ca}^{2+}$ because the diffusional exchange between $\mathrm{Na}^{+}$and $\mathrm{NH}_{4}{ }^{+}$was reduced by $35 \%$.

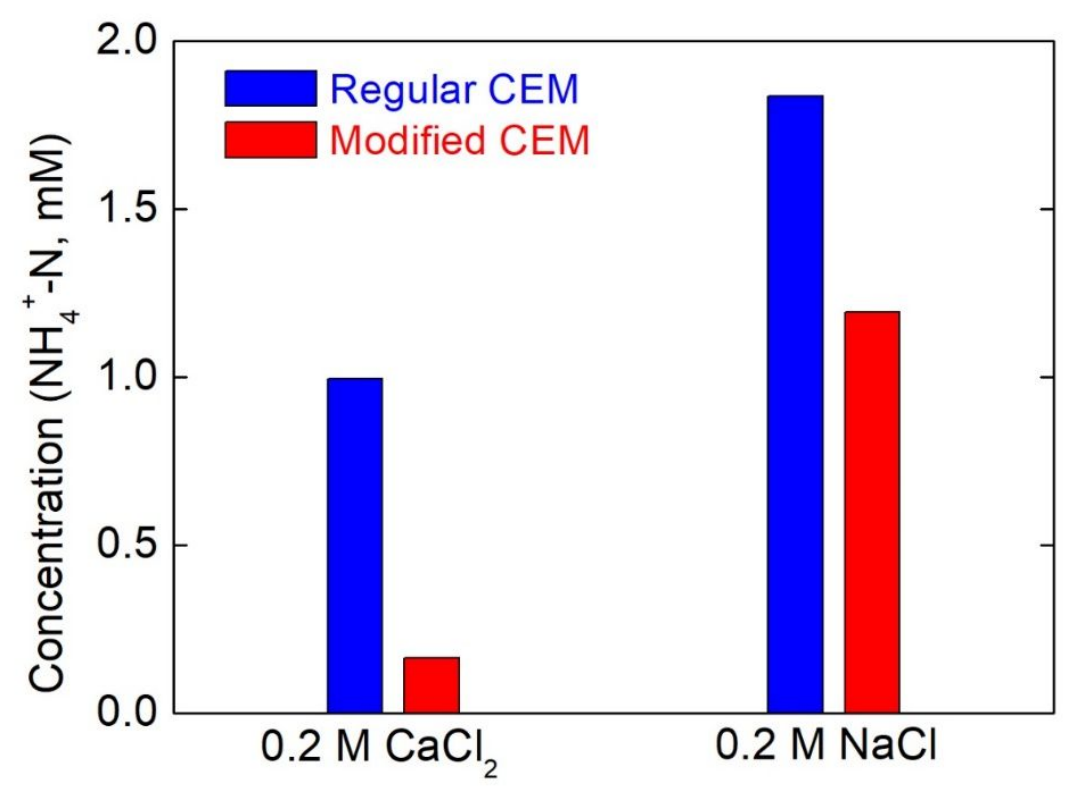

Figure S7. Concentration of $\mathrm{NH}_{4}{ }^{+}$in $0.2 \mathrm{M} \mathrm{CaCl}_{2}$ or $0.2 \mathrm{M} \mathrm{NaCl}$ solution after diffusional exchange from synthetic wastewater $\left(20 \mathrm{mM} \mathrm{NaCl}+5 \mathrm{mM} \mathrm{NH}_{4} \mathrm{Cl}\right)$ using 2-channel flow cell. 


\section{Supporting Information Note 2. Separation performance using regular CEMs}

The use of regular CEM produced a nitrogen flux of $11.8 \mathrm{~g} \mathrm{~N} \mathrm{~m}^{-2} \mathrm{~d}^{-1}$ without applied current due to the diffusional exchange between $\mathrm{NH}_{4}{ }^{+}$(central channel) and $\mathrm{Ca}^{2+}$ (anode and cathode channels). As shown in Figure S8a, the zero current nitrogen flux was significant compared to fluxes at 4.8 $\mathrm{A} \mathrm{m}^{-2}\left(15.9 \mathrm{~g} \mathrm{~N} \mathrm{~m}^{-2} \mathrm{~d}^{-1}\right), 9.6 \mathrm{~A} \mathrm{~m}^{-2}\left(18.2 \mathrm{~g} \mathrm{~N} \mathrm{~m}^{-2} \mathrm{~d}^{-1}\right)$, and $19.2 \mathrm{~A} \mathrm{~m}^{-2}\left(28.4 \mathrm{~g} \mathrm{~N} \mathrm{~m}^{-2} \mathrm{~d}^{-1}\right)$. The undesired distribution of $\mathrm{NH}_{4}{ }^{+}$in cathode and anode channels was dominant regardless of current densities (Figure S8b), which complicated the analysis for calculating nitrogen flux and energy consumption contributed by the redox couple.

(a)

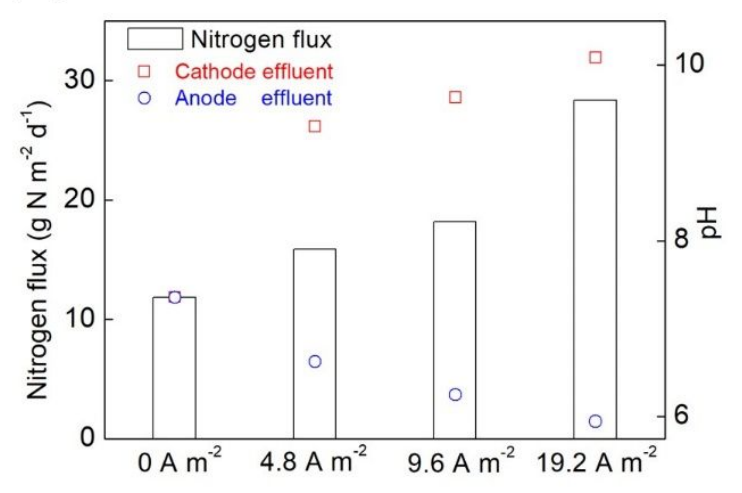

(b)

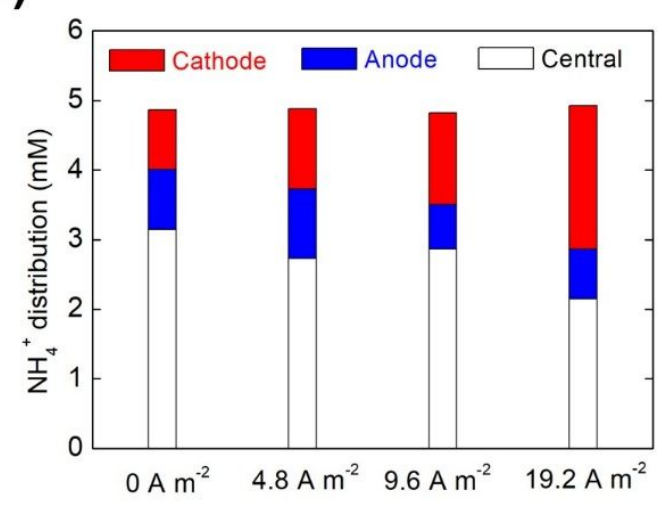

Figure S8. (a) Nitrogen flux and effluent $\mathrm{pH}$ as a function of current density. Electrolyte (15 mM $\mathrm{HQ} / \mathrm{BQ}$ and $0.2 \mathrm{M} \mathrm{CaCl}_{2}$ ) and synthetic wastewater $\left(20 \mathrm{mM} \mathrm{NaCl}\right.$ and $5 \mathrm{mM} \mathrm{NH} \mathrm{Nl}_{4} \mathrm{Cl}$ ) were circulated through each channel without recycle at $0.5 \mathrm{~mL} \mathrm{~min}^{-1}$. (b) Distribution of ammonium in effluents from cathode, anode, and central channels as function of current density. 


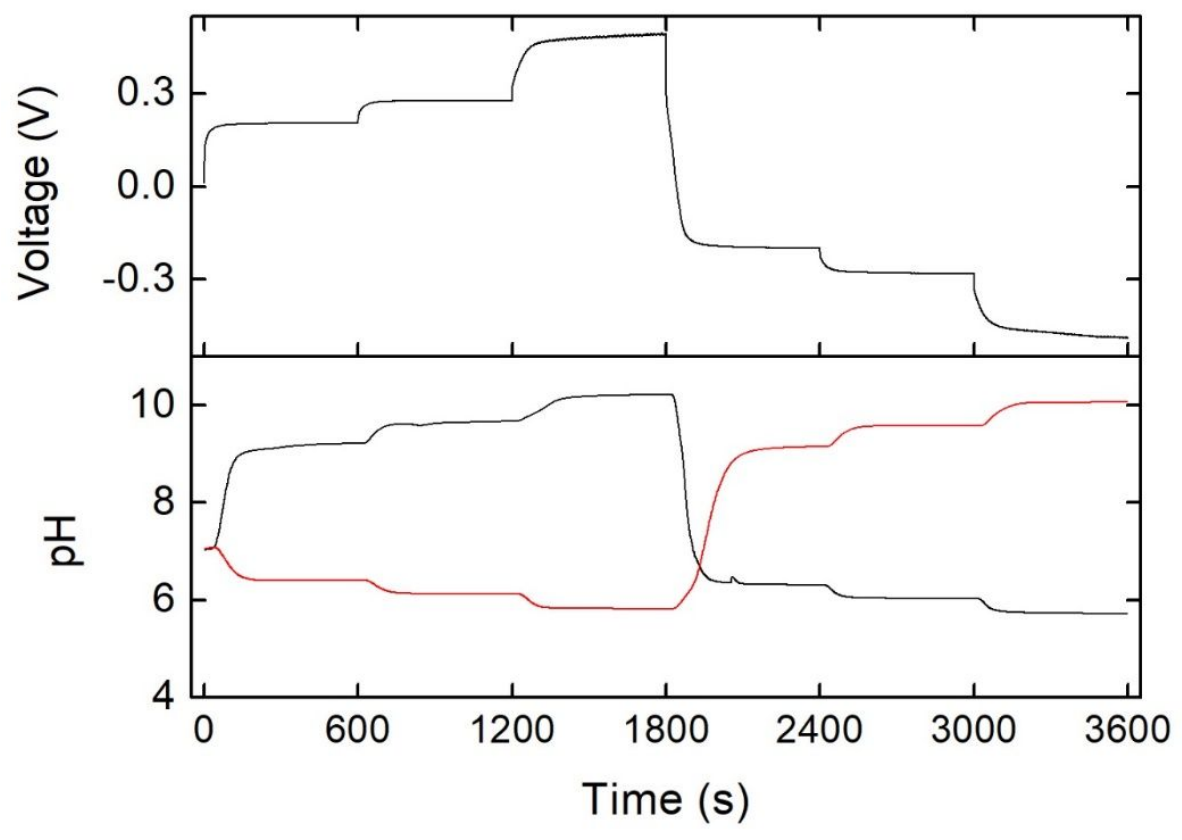

Figure S9. Voltage and effluent $\mathrm{pH}$ profiles recorded at $\pm 3.5,7.0$, and $14 \mathrm{~mA}$ in the 2-channel flow cell at a flow rate of $0.5 \mathrm{~mL} \mathrm{~min}^{-1}$ by feeding each channel using $15 \mathrm{mM} \mathrm{HQ} / \mathrm{BQ}+0.2 \mathrm{M}$ CaAc. 

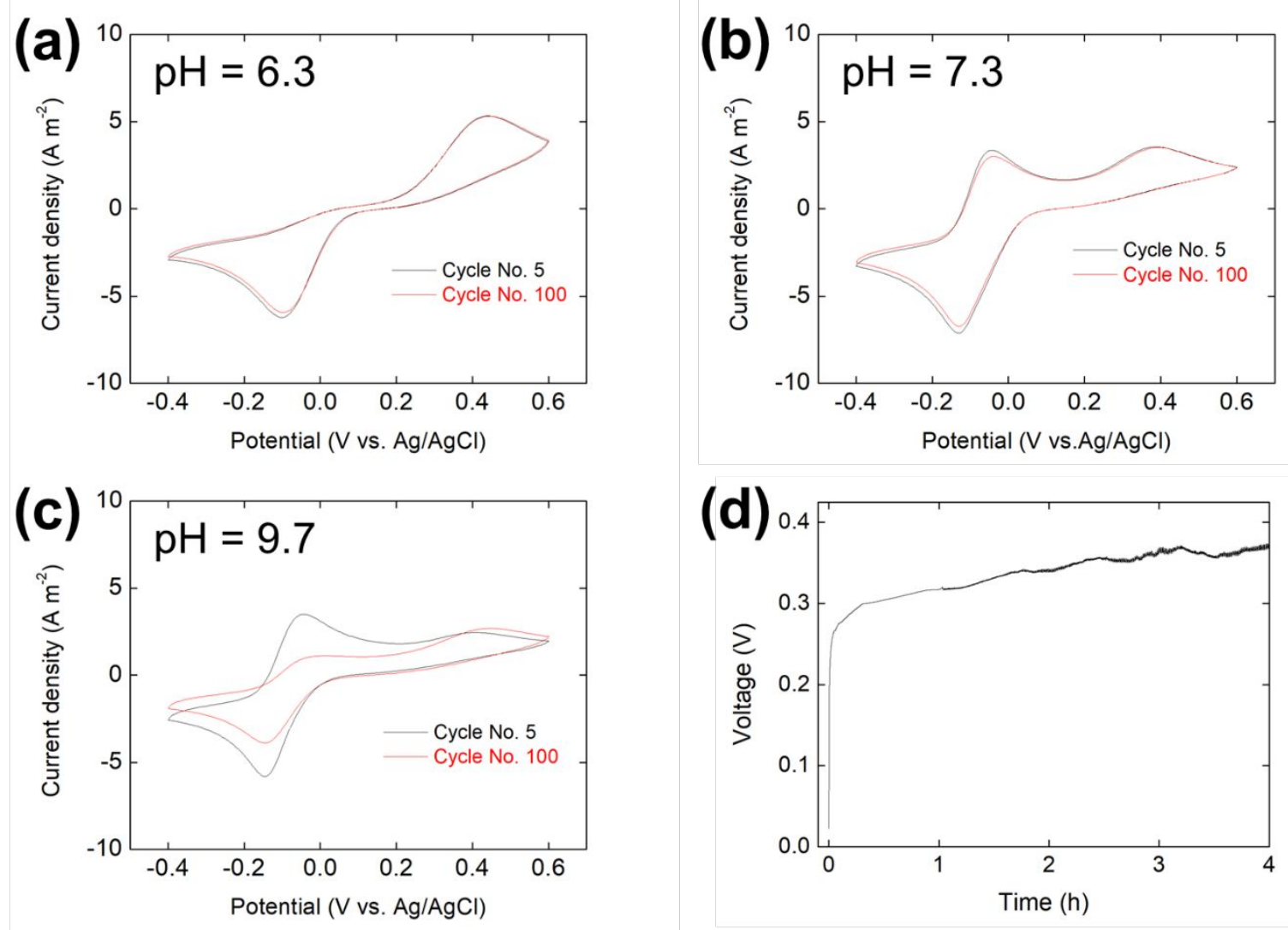

Figure S10. Cyclic voltammetry profiles of electrolytes (1 mM HQ/BQ $+0.2 \mathrm{M} \mathrm{CaAc})$ at (a) $\mathrm{pH}$ 6.3 , (b) 7.3, and (c) 9.7 (scan rate $=100 \mathrm{mV} \mathrm{s}^{-1}$ ). Cycling tests were performed under conditions representing $\mathrm{pH}$ decrease $(\mathrm{pH}=6.3)$, initial $(\mathrm{pH}=7.3)$, and $\mathrm{pH}$ increase $(\mathrm{pH}=9.7)$. (d) Cell voltage recorded while recirculating $100 \mathrm{~mL}$ of redox electrolyte $(15 \mathrm{mM} \mathrm{HQ} / \mathrm{BQ}$ in $0.2 \mathrm{M} \mathrm{CaAc})$ through a 2-channel flow cell divided by a cation exchange membrane at a flow rate of $0.5 \mathrm{~mL} \mathrm{~min}^{-1}$ for 4 hours. The basic condition was detrimental to the stability of $\mathrm{HQ} / \mathrm{BQ}$, which was evidenced by current decay in 100 cycles (c) as well as the gradual increase in the cell voltage (d). 
Table S1. Comparison of electrochemical techniques to remove and recover ammonia from wastewater.

\begin{tabular}{|c|c|c|c|c|c|c|}
\hline Methods & $\begin{array}{c}\text { Ammonia } \\
\text { Concentration } \\
(\mathrm{mM})\end{array}$ & $\begin{array}{l}\text { Current Density } \\
\qquad\left(\mathrm{A} \mathrm{m}^{-2}\right)\end{array}$ & $\begin{array}{c}\text { Ammonia } \\
\text { Removal/Recovery }\end{array}$ & $\begin{array}{l}\text { Nitrogen Flux } \\
\left(\mathrm{g} \mathrm{N} \mathrm{m}^{-2} \mathrm{~d}^{-1}\right)\end{array}$ & $\begin{array}{c}\text { Energy } \\
\text { Consumption }(\mathrm{kWh} \\
\left.\mathrm{kg}^{-1} \mathrm{~N}\right)\end{array}$ & References \\
\hline Haber-Bosch & & & & & 10.3 & 3 \\
\hline $\begin{array}{l}\text { ED with membrane } \\
\text { contactor }\end{array}$ & 535 & 100 & $\begin{array}{l}60 \text { (removal) } \\
30 \text { (recovery) }\end{array}$ & $\begin{array}{l}1710 \text { (removal) } \\
1010 \text { (recovery) }\end{array}$ & 8.5 (recovery) & 4 \\
\hline $\begin{array}{l}\text { ED with column } \\
\text { stripping }\end{array}$ & 364 & 40 & $\begin{array}{l}75 \text { (removal) } \\
58 \text { (recovery) }\end{array}$ & 235 (recovery) & 14.7 (recovery) & 5 \\
\hline $\begin{array}{l}\text { ED with column } \\
\text { stripping }\end{array}$ & 151 & 30 & 63 (removal) & 142 (removal) & 26 (removal) & 6 \\
\hline $\begin{array}{l}\text { ED with membrane } \\
\text { stripping }\end{array}$ & 142 & 50 & 63 (removal) & 335 (removal) & 13.6 (removal) & 7 \\
\hline $\begin{array}{c}\mathrm{H}_{2} \text { recycling } \\
\text { electrochemical } \\
\text { system }\end{array}$ & 243 & 20 & 73 (removal) & 151 (removal) & 7.3 (removal) & 8 \\
\hline MCDI & 7.5 & $2 \mathrm{~V}^{\mathrm{b}}$ & 98 (removal) & $\mathrm{N} / \mathrm{A}^{\mathrm{a}}$ & $\mathrm{N} / \mathrm{A}^{\mathrm{a}}$ & 9 \\
\hline MCDI with BMED & 12.5 & 200 & 77 (removal) & $\mathrm{N} / \mathrm{A}^{\mathrm{a}}$ & 3.2 (MCDI) & 10 \\
\hline $\begin{array}{c}\text { BMED with } \\
\text { membrane stripping }\end{array}$ & 14.3 & 19 & 65.2 (removal) & $\mathrm{N} / \mathrm{A}^{\mathrm{a}}$ & $1.3(\text { removal })^{\mathrm{d}}$ & 11 \\
\hline $\begin{array}{c}\text { BMED with } \\
\text { membrane stripping }\end{array}$ & 111.1 & 100 & 80 (removal) & 819 (removal) & 5.1 (removal) $^{\mathrm{e}}$ & 12 \\
\hline Two-stage BMED & 300 & 150 & 78 (removal) & $\mathrm{N} / \mathrm{A}^{\mathrm{a}}$ & $\mathrm{N} / \mathrm{A}^{\mathrm{a}}$ & 13 \\
\hline BDI & 3.4 & $0.2 \mathrm{~V}^{\mathrm{b}}$ & 85 (removal) & $9.9^{c}$ & 1.5 (removal) & 14 \\
\hline $\begin{array}{c}\text { Flow-electrode } \\
\text { MCDI with } \\
\text { membrane stripping }\end{array}$ & 2.9 & 11.5 & $\begin{array}{l}90 \text { (removal) } \\
55 \text { (recovery) }\end{array}$ & $\begin{array}{l}25.3 \text { (removal) } \\
16.5 \text { (recovery) }\end{array}$ & 21.7 (recovery) & 15 \\
\hline Flow-electrode & 3 & 10.4 & 80 (removal) & 16.9 (recovery) & 48.8 (recovery) & 16 \\
\hline
\end{tabular}




\begin{tabular}{|c|c|c|c|c|c|c|}
\hline $\begin{array}{c}\text { MCDI with } \\
\text { membrane stripping }\end{array}$ & & & 62 (recovery) & & & \\
\hline $\begin{array}{c}\text { Flow electrode } \\
\text { MCDI }\end{array}$ & 3 & 6 & 70 (removal) & $\mathrm{N} / \mathrm{A}^{\mathrm{a}}$ & 35.4 (removal) & 17 \\
\hline $\begin{array}{l}\text { ED with membrane } \\
\text { stripping }\end{array}$ & 2.14 & 100 & $\begin{array}{l}99 \text { (removal) } \\
54 \text { (recovery) } \\
99 \text { (recovery) }\end{array}$ & $\begin{array}{c}337(\text { removal })^{c} \\
32.7(54 \% \text { recovery })^{c} \\
13.4(99 \% \text { recovery })^{c}\end{array}$ & $\mathrm{~N} / \mathrm{A}^{\mathrm{a}}$ & 18 \\
\hline $\begin{array}{l}\text { Proton-mediated } \\
\text { redox couple with } \\
\text { membrane stripping }\end{array}$ & 5 & 9.6 & $\begin{array}{l}50 \text { (removal) } \\
18 \text { (recovery) }\end{array}$ & $\begin{array}{l}34.9 \text { (removal) } \\
12.4 \text { (recovery) }\end{array}$ & $\begin{array}{l}3.2 \text { (removal) } \\
9.1 \text { (recovery) }\end{array}$ & This study \\
\hline \multicolumn{7}{|c|}{ 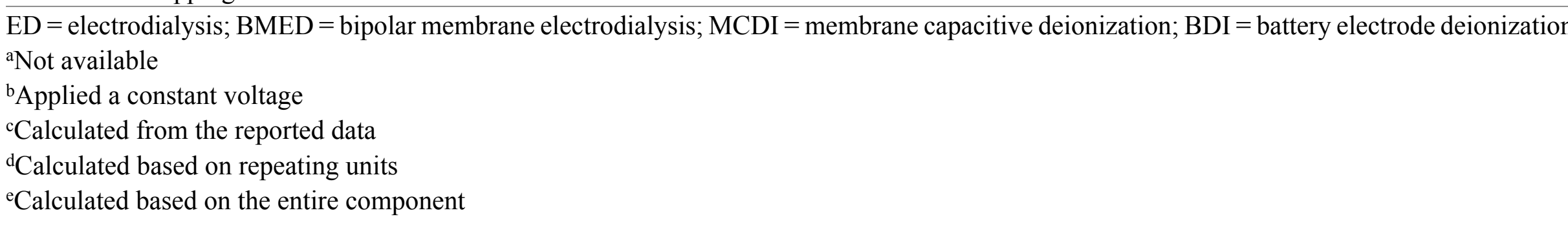 } \\
\hline
\end{tabular}




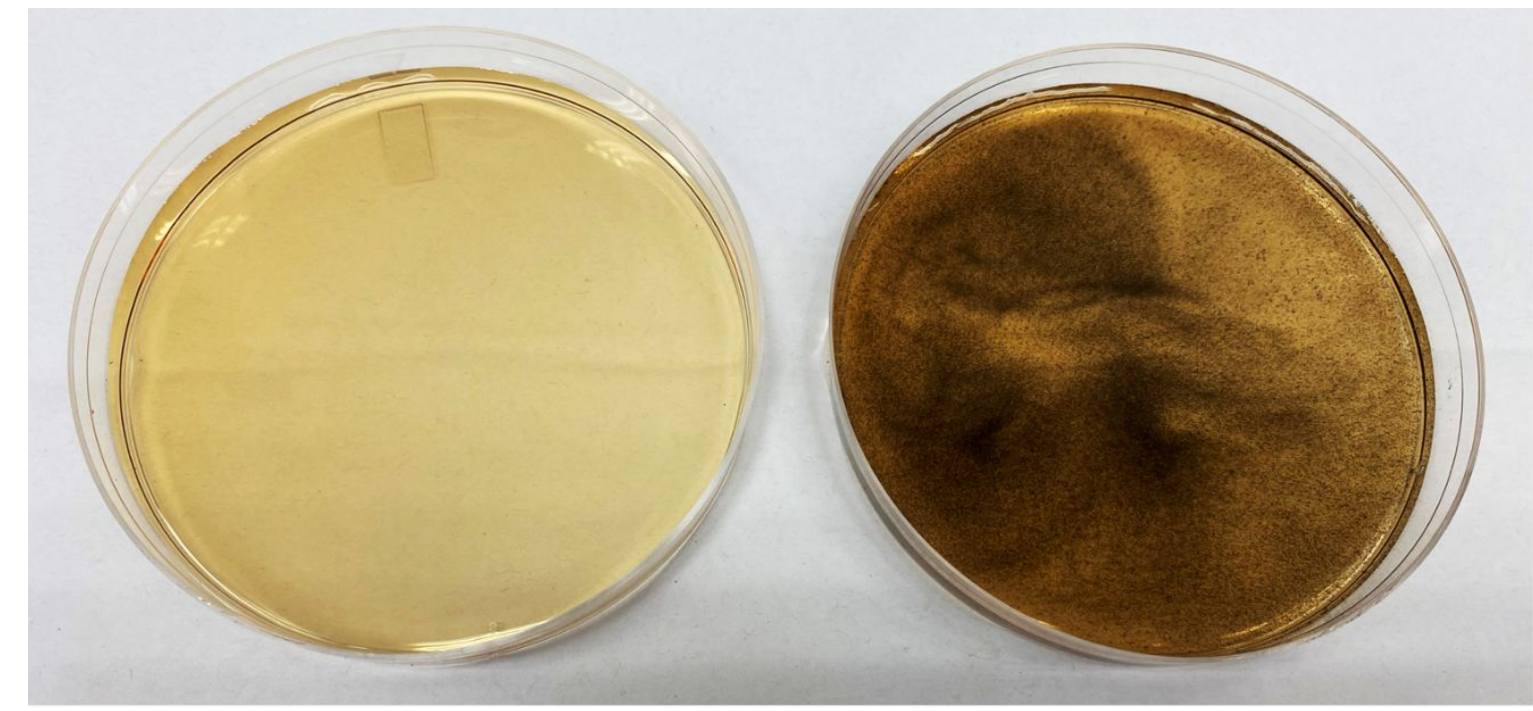

$15 \mathrm{mM} \mathrm{HQ/BQ}$ in $0.2 \mathrm{M} \mathrm{CaAc} \quad 30 \mathrm{mM} \mathrm{HQ/BQ}$ in $0.2 \mathrm{M} \mathrm{CaAc}$

Figure S11. Photo of solutions containing $15 \mathrm{mM} \mathrm{HQ} / \mathrm{BQ}$ in $0.2 \mathrm{M} \mathrm{CaAc}$ (left) and $30 \mathrm{mM}$ $\mathrm{HQ} / \mathrm{BQ}$ in $0.2 \mathrm{M} \mathrm{CaAc}$ (right), indicating that the HQ/BQ redox couple is not soluble at the concentration of $30 \mathrm{mM}$. 


\section{References}

(1) White, N.; Misovich, M.; Yaroshchuk, A.; Bruening, M. L. Coating of Nafion Membranes with Polyelectrolyte Multilayers to Achieve High Monovalent/Divalent Cation Electrodialysis Selectivities. ACS Appl. Mater. Inter. 2015, 7, 6620-6628.

(2) Xi, J.; Wu, Z.; Teng, X.; Zhao, Y.; Chen, L.; Qiu, X. Self-Assembled Polyelectrolyte Multilayer Modified Nafion Membrane with Suppressed Vanadium Ion Crossover for Vanadium Redox Flow Batteries. J. Mater. Chem. 2008, 18, 1232-1238.

(3) Maurer, M.; Schwegler, P.; Larsen, T. A. Nutrients in Urine: Energetic Aspects of Removal and Recovery. Water Sci. Technol. 2003, 48, 37-46.

(4) Tarpeh, W. A.; Barazesh, J. M.; Cath, T. Y.; Nelson, K. L. Electrochemical Stripping to Recover Nitrogen from Source-Separated Urine. Environ. Sci. Technol. 2018, 52, 14531460.

(5) Luther, A. K.; Desloover, J.; Fennell, D. E.; Rabaey, K. Electrochemically Driven Extraction and Recovery of Ammonia from Human Urine. Water Res. 2015, 87, 367-377.

(6) Desloover, J.; Abate Woldeyohannis, A.; Verstraete, W.; Boon, N.; Rabaey, K. Electrochemical Resource Recovery from Digestate to Prevent Ammonia Toxicity during Anaerobic Digestion. Environ. Sci. Technol. 2012, 46, 12209-12216.

(7) Rodríguez Arredondo, M.; Kuntke, P.; ter Heijne, A.; Hamelers, H. V. M.; Buisman, C. J. N. Load Ratio Determines the Ammonia Recovery and Energy Input of an Electrochemical System. Water Res. 2017, 111, 330-337.

(8) Kuntke, P.; Rodríguez Arredondo, M.; Widyakristi, L.; ter Heijne, A.; Sleutels, T. H. J. A.; Hamelers, H. V. M.; Buisman, C. J. N. Hydrogen Gas Recycling for Energy Efficient Ammonia Recovery in Electrochemical Systems. Environ. Sci. Technol. 2017, 51, 3110 3116.

(9) Wimalasiri, Y.; Mossad, M.; Zou, L. Thermodynamics and Kinetics of Adsorption of Ammonium Ions by Graphene Laminate Electrodes in Capacitive Deionization. Desalination 2015, 357, 178-188.

(10) Gao, F.; Wang, L.; Wang, J.; Zhang, H.; Lin, S. Nutrient Recovery from Treated Wastewater by a Hybrid Electrochemical Sequence Integrating Bipolar Membrane Electrodialysis and Membrane Capacitive Deionization. Environ. Sci. Water Res. Technol. 2020, 6, 383-391.

(11) Yan, H.; Wu, L.; Wang, Y.; Shehzad, M. A.; Xu, T. Ammonia Capture by Water Splitting and Hollow Fiber Extraction. Chem. Eng. Sci. 2018, 192, 211-217.

(12) Rodrigues, M.; de Mattos, T. T.; Sleutels, T.; ter Heijne, A.; Hamelers, H. V. M.; Buisman, C. J. N.; Kuntke, P. Minimal Bipolar Membrane Cell Configuration for Scaling Up Ammonium Recovery. ACS Sustain. Chem. Eng. 2020, 8, 17359-17367.

(13) Shi, L.; Hu, Y.; Xie, S.; Wu, G.; Hu, Z.; Zhan, X. Recovery of Nutrients and Volatile Fatty Acids from Pig Manure Hydrolysate Using Two-Stage Bipolar Membrane Electrodialysis. Chem. Eng. J. 2018, 334, 134-142.

(14) Kim, T.; Gorski, C. A.; Logan, B. E. Ammonium Removal from Domestic Wastewater Using Selective Battery Electrodes. Environ. Sci. Technol. Lett. 2018, 5, 578-583.

(15) Zhang, C.; Ma, J.; He, D.; Waite, T. D. Capacitive Membrane Stripping for Ammonia Recovery (CapAmm) from Dilute Wastewaters. Environ. Sci. Technol. Lett. 2018, 5, 43- 
49.

(16) Zhang, C.; Ma, J.; Song, J.; He, C.; Waite, T. D. Continuous Ammonia Recovery from Wastewaters Using an Integrated Capacitive Flow Electrode Membrane Stripping System. Environ. Sci. Technol. 2018, 52, 14275-14285.

(17) Zhang, C.; Ma, J.; Waite, T. D. Ammonia-Rich Solution Production from Wastewaters Using Chemical-Free Flow-Electrode Capacitive Deionization. ACS Sustain. Chem. Eng. 2019, 7, 6480-6485.

(18) Liu, M. J.; Neo, B. S.; Tarpeh, W. A. Building an Operational Framework for Selective Nitrogen Recovery via Electrochemical Stripping. Water Res. 2020, 169, 115226. 